Kansas State University Libraries

New Prairie Press

\title{
A BOOTSTRAP ANALYSIS OF TEMPERATURE EFFECTS ON BEAN LEAF BEETLE EGG HATCH TIMES
}

Kenneth J. Koehler

Follow this and additional works at: https://newprairiepress.org/agstatconference

Part of the Agriculture Commons, and the Applied Statistics Commons

\section{(c) (1) $\Theta($}

This work is licensed under a Creative Commons Attribution-Noncommercial-No Derivative Works 4.0 License.

\section{Recommended Citation}

Koehler, Kenneth J. (1994). "A BOOTSTRAP ANALYSIS OF TEMPERATURE EFFECTS ON BEAN LEAF BEETLE EGG HATCH TIMES," Conference on Applied Statistics in Agriculture. https://doi.org/10.4148/ 2475-7772.1361

This is brought to you for free and open access by the Conferences at New Prairie Press. It has been accepted for inclusion in Conference on Applied Statistics in Agriculture by an authorized administrator of New Prairie Press. For more information, please contact cads@k-state.edu. 


\title{
A BOOTSTRAP ANALYSIS OF TEMPERATURE EFFECTS ON BEAN LEAF BEETLE EGG HATCH TIMES
}

\author{
Kenneth J. Koehler \\ Department of Statistics \\ Iowa State University, Ames, Iowa 51011
}

\begin{abstract}
The bean leaf beetle (Cerotoma trifurcata) is a significant soybean pest in the Midwest. The possibility of reducing crop damage by disrupting the synchrony between emergence of F2 adults and the availability of young green pods requires an increased understanding of bean leaf beetle (BLB) phenology. An important consideration in predicting emergence of F2 adults is the influence of temperature on the rates of egg and larval development. In this article we concentrate on the analysis of data from a controlled study of the effects of temperature on both the hatch time distribution and the viability of BLB eggs. Several temperature levels are considered with a different number of egg cohorts exposed to each of several temperature levels. Hatch time observations for individual eggs are subject to both interval and right censoring, and inspection schedules vary across cohorts. Limited failure population (LFP) models with Weibull hatch time distributions are used to estimate parameters of the hatch time distribution and the proportion of viable eggs at each temperature. These estimates are used in the subsequent weighted least squares estimation of curves for predicting the proportion of viable eggs and the inverse median of the hatch time distribution as functions of temperature. Bootstrap procedures are used to estimate variances that properly account for within cohort correlations. The importance of replicating experiments for different temperature levels is illustrated.
\end{abstract}

Key Words: Bootstrap estimation, censored data, limited failure survival models.

\section{Introduction}

In Iowa, two generations of BLB adults develop each year (Smelser and Pedigo, 1991). Overwintered BLB adults colonize soybean fields soon after seedling emergence and lay eggs which develop into F1 adults. Because F1 adults are usually present while soybeans are still in vegetative growth stages, feeding by F1 adults is usually confined to foliage, resulting in little if any yield reduction (Smelser and Pedigo, 1992). Eggs laid by the F1 adults develop into F2 adults which typically emerge in early August when soybeans are in growth stage R5. F2 adults supplement their leaf diet with outer green tissues of stems, peduncles, and pods. Since BLB adults prefer green immature pods to dry mature pods, the degree to which feeding by $\mathrm{F} 2$ adults reduces yield depends on the 
relative phenologies of the beetles and the crop. Disrupting the synchrony between emergence of F2 adults and availability of green pods is a promising BLB management strategy that requires an increased understanding of BLB phenology. An important consideration in predicting the emergence of $\mathrm{F} 2$ adults is the influence of temperature on egg and larval development rates (e.g. Herzog et al. 1974 and Turner 1979). This article is restricted to the analysis of temperature effects on egg hatch times and viability, but the basic method could be equally well applied to the analysis of various stages of larval development to analyze the effects of temperature on total development time from egg deposit to the emergence of F2 adults.

To quantify the effects of temperature on the viability and developmental velocity of BLB eggs, multiple cohorts of eggs were incubated at specific temperatures. Each cohort consisted about 20 eggs laid in the same day and incubated in a specific rearing cup. In this case, existence of within cohort correlations deviates from the usual assumption made in survival and life event analysis that each response time is independent of any other response time (e.g. Cox and Oakes, 1984, or Kalbfleisch and Prentice, 1980). Variation in conditions within a single temperature chamber results in a tendency for responses from eggs within a single rearing cup to be more similar than responses from eggs in other cups incubated at the same temperature. Additional correlations arise from genetic similarities of eggs laid by a single beetle, but eggs laid by individual adult females could not be identified because eggs were collected from cages containing more than one adult female. Consequently, unequal correlations among eggs in a single cohort arising from eggs laid by the same or different beetles cannot be directly modelled. Bootstrap resampling procedures are used to obtain standard errors for estimates of parameters in the temperature curves that properly account for effects of within cohort correlations.

An additional complication is that some eggs are not viable, i.e., they never hatch, and the proportion of viable eggs could be affected by temperature. Due to varying inspection schedules, final inspection times are not the same for all egg cohorts, and some viable eggs may not be observed to hatch prior to the final inspection times for their respective cohorts. A limited failure population (LFP) approach is used to allow for this possibility. The results indicate, however, that few eggs hatched after the final inspection times.

\section{Experimental Procedures}

Eggs were collected from BLB adults reared at $30^{\circ} \mathrm{C}$ under constant daily photoperiods consisting of 16 hours of light and 8 hours of darkness. Cohorts, consisting of about 20 eggs, collected on the same day and placed in a single rearing cup, were randomly assigned to incubation at one of the prescribed temperatures. Multiple cohorts were incubated at the same temperature in a single temperature chamber. Each egg was collected on the day it was laid, and the same photoperiod of 16 hours of light per day was used for all egg cohorts. In most cases, all eggs in a single rearing cup (cohort) 
were obtained from a single cage of adults, but achieving the goal of about 20 eggs per cup sometimes made it necessary to put eggs from different cages into a single cup. Each cage contributed eggs to only one cohort. Numbers of eggs varied among cohorts, but eighty percent of the cohorts contained between 18 and 22 eggs. The extremes were 4 eggs in one cup and 38 eggs in another. Hatch times were recorded for individual eggs. Results for eggs in different cohorts are assumed to be independent, but there are substantial correlations among results for eggs within individual cohorts. Within cohort correlations need not be homogeneous across cohorts nor even for different pairs of eggs in any single cohort.

Hatch times were recorded for individual eggs as the number of days after the eggs were laid with the day on which the egg was laid taken to be day 0. Although the time of day was kept reasonably constant, the set of days on which inspections were done varied across cohorts, and no cohort was inspected more than once on any single day. Consequently, hatch times are interval censored with the shortest inspection intervals corresponding to a single day and other inspection intervals consisting of two or more days. The data for 20 of the $111 \mathrm{egg}$ cohorts incubated at $25^{\circ} \mathrm{C}$ in the first year of the study are displayed in Table 1. Data are not displayed for the first 6 days after eggs were laid because no eggs were observed to hatch during that period. Dots represent days on which inspections were not made. The value 4 at day 8 for cohort 1 , for example, indicates that 4 eggs hatched between 6 and 8 days after the eggs were laid, and the value 11 at day 10 indicates that 11 eggs hatched between 9 and 10 days after the eggs were laid. Since this cohort was not inspected after day 10, hatch times for any eggs that hatched after day 10 are right censored. These data provide a clear indication of the variation in inspection times among egg cohorts. The observed numbers of viable eggs, reported in the last column of Table 2, provide lower bounds on the actual numbers of viable eggs in the individual cohorts.

\section{Hatch Time Model}

For a particular temperature, the data consist of observations on individual eggs in $m$ independent cohorts where the hatch times and censoring times for the $n_{i}$ eggs in the $\mathrm{i}$-th cohort fall into $\mathrm{K}(\mathrm{i})+1$ disjoint time intervals, $\left\{\left[\mathrm{t}_{\mathrm{i}, \mathrm{k}-1}, \mathrm{t}_{\mathrm{i}, \mathrm{k}}\right): \mathrm{k}=1,2, \ldots, \mathrm{K}(\mathrm{i})+1\right\}$, determined by the inspection schedule for that particular cohort. By convention, $\mathrm{t}_{\mathrm{i}, 0}=0$, $t_{i, K(i)+1}=\infty$ and $t_{i, K(i)}$ is the last inspection time for the $\mathrm{i}$-th cohort. The survivor function $\mathrm{S}(\mathrm{t})$ is the conditional probability that an egg does not hatch before time $\mathrm{t}$, given that it is a viable egg. Denoting the probability of a viable egg by $\pi$, the unconditional probability that a randomly selected egg hatches in time interval $\left[\mathrm{t}_{\mathrm{i}, \mathrm{k}}, \mathrm{t}_{\mathrm{i}, \mathrm{k}+1}\right)$ is

$$
\pi\left[\mathrm{S}\left(\mathrm{t}_{\mathrm{i}, \mathrm{k}-1}\right)-\mathrm{S}\left(\mathrm{t}_{\mathrm{i}, \mathrm{k}}\right)\right]
$$

and the unconditional probability that a randomly selected egg does not hatch before the final inspection time is 


$$
(1-\pi)+\pi \mathrm{S}\left(\mathrm{t}_{\mathrm{i}, \mathrm{K}(\mathrm{i})}\right)
$$

the sum of the probability of randomly selecting a nonviable egg and the probability of selecting a viable egg that does not hatch before the final inspection time. Meeker (1987) and Koehler and McGovern (1990) refer to this as a limited failure population (LFP) model in applications where failure times are observed, but it can also be used to model life event times such as the egg hatch times considered in this study.

Estimates of $\pi$ and parameters in the survivor function are obtained by maximizing

$$
\mathrm{L}=\prod_{\mathrm{i}=1}^{\mathrm{m}}\left\{(1-\pi)+\pi \mathrm{S}\left(\mathrm{t}_{\mathrm{i}, \mathrm{K}(\mathrm{i})}\right)\right\}^{\mathrm{c}_{\mathrm{K}(\mathrm{i})}} \prod_{\mathrm{k}=1}^{\mathrm{K}(\mathrm{i})}\left\{\pi\left[\mathrm{S}\left(\mathrm{t}_{\mathrm{i}, \mathrm{k}-1}\right)-\mathrm{S}\left(\mathrm{t}_{\mathrm{i}, \mathrm{k}}\right)\right]\right\}, \mathrm{n}_{\mathrm{ik}}
$$

where $n_{i k}$ is the number of eggs in the $\mathrm{i}$-th cohort that hatch in the $\mathrm{k}$-th inspection interval for that cohort and $\mathrm{c}_{\mathrm{K}(\mathrm{i})}$ is the number of eggs in the $\mathrm{i}$-th cohort that are right censored at the final inspection time for that cohort. Although (3) is proportional to the likelihood function for a model that incorrectly assumes that each egg responds independently of any other egg, the resulting estimators are consistent when $S(t)$ is correctly specified. Huster, et al (1989) refer to this as an independence working model (IWM) approach. Unlike the situation where the correct likelihood is maximized, the inverse of the information matrix obtained from the second partial derivatives of the logarithm of (3) is generally not a consistent estimator of the covariance matrix of the parameter estimates. Consequently, we use bootstrap resampling to obtain consistent estimates of covariance matrices.

Preliminary analyses of the observed hatch times indicated that two-parameter Weibull distributions with different starting times provide adequate models for $\mathrm{S}(\mathrm{t})$ for the three incubation temperatures. A different set of parameters is estimated for each temperature level. The survivor function for a two-parameter Weibull distribution with known starting time $\mathrm{A}$ is

$$
\mathrm{S}(\mathrm{t})=\exp \left\{-[(\mathrm{t}-\mathrm{A}) / \delta]^{\gamma}\right\}, \quad \mathrm{t}>\mathrm{A}, \delta>0, \gamma>0
$$

and the median hatch time is

$$
\mathrm{M}=\mathrm{A}+\delta[\ln (2)]^{1 / \gamma}
$$

The inverse of the median hatch time is often used as an indicator of egg development rate. Different starting times are used to indicate the earliest possible hatch dates at different incubation temperatures.

Since the LFP model allows for the possibility that viable eggs may hatch after the final inspection time, some care must used in its application. Early termination of 
inspection results in a large proportion of unhatched eggs at the final inspection time and unreliable estimation of model parameters. When inspection is terminated too early, the IWM likelihood (3) is quite flat in a large region containing the maximum, because it is not clear whether (2) should be made large by using a small value of $\pi$ or by using a large value of $\pi$ and adjusting the parameters in the survivor function to make $S(t)$ large at the final inspection time. Based on a Monte Carlo study of the case of completely independent responses, Meeker (1987) recommends that inspection should continue until at least 80 percent of the eventual failures (hatches of viable eggs) have occurred. Unpublished simulations we performed indicate that this is also a reasonable guideline when responses are correlated. Premature termination of inspection tends to result in estimates of $\pi$ that are too close to 1 . In the present study, the final inspection times were selected so that nearly all of the viable eggs would be observed to hatch and the $80 \%$ guideline is clearly satisfied.

\section{Bootstrap Estimation}

It follows from Huber (1967) that maximization of $L$ in (3) produces consistent parameter estimates with large sample normal distributions. The inverse of the matrix of second partial derivatives of $\log (\mathrm{L})$, however, yields variance and covariance estimates that tend to be too small in the presence of positive intra-cohort correlations. More accurate estimates of covariance matrices and confidence intervals with appropriate coverage probabilities are obtained from a bootstrap procedure that approximates the selection of new samples from the population by sampling with replacement from the original sample. Efron $(1979,1982)$ reviews and develops basic theory underlying the bootstrap and other resampling procedures for data obtained from simple random samples. The BLB egg hatch study is somewhat different because it involves cohorts of eggs with possible intra-cohort correlations. Following the approach used by Koehler and McGovern (1990), the bootstrap procedure is applied by using cohorts as the basic sampling units, rather than individual eggs.

Consider hatch time data for $\mathrm{m}$ cohorts of BLB eggs incubated at a particular temperature. Each iteration of the bootstrap procedure mimics a new replication of a study of hatch times for $m$ cohorts of BLB eggs maintained at the same temperature. This is done as follows:

(a) Use simple random sampling with replacement to select a sample of $\mathrm{m}$ cohorts from the original set of $m$ cohorts.

(b) Maximize (3) to obtain parameter estimates for the Weibull LFP model, say $\hat{\delta}, \hat{\gamma}$, and $\hat{\pi}$, from the bootstrapped sample from step (a).

(c) Repeat steps (a) and (b) B times, where B represents the total number of bootstrapped samples. In the present analysis, $\mathrm{B}=1000$. 
This produces B independent sets of parameter estimates, $\hat{\boldsymbol{\beta}}_{\mathrm{i}}=\left(\hat{\delta}_{\mathrm{i}}, \hat{\gamma}_{\mathrm{i}}, \hat{\pi}_{\mathrm{i}}\right)^{\mathrm{T}}, \mathrm{i}=1,2, \ldots, \mathrm{B}$, one set for each of the $\mathrm{B}$ bootstrapped samples. Distributional properties of these $\mathrm{B}$ estimates are used to approximate the corresponding properties of the parameter estimates computed from the original sample. For example, the sample covariance matrix for the parameter estimates evaluated from the original data is estimated as

$$
\mathrm{V}=(\mathrm{B}-1)^{-1} \sum_{\mathrm{i}=1}^{\mathrm{B}}\left(\hat{\boldsymbol{\beta}}_{\mathrm{i}}-\hat{\boldsymbol{\beta}}_{+}\right)\left(\hat{\boldsymbol{\beta}}_{\mathrm{i}}-\hat{\boldsymbol{\beta}}_{+}\right)^{\mathrm{T}}
$$

where $\hat{\beta}_{+}=\sum_{\mathrm{i}} \hat{\beta}_{\mathrm{i}} / \mathrm{B}$.

Since resampling of egg cohorts is done with replacement with probability $1 / \mathrm{m}$ assigned to each of the $\mathrm{m}$ cohorts in the original sample, any particular bootstrapped sample of $m$ cohorts may contain multiple copies of some egg cohorts and no copies of other egg cohorts from the original sample. Bootstrapped samples need not contain the same number of individual eggs as the original sample, and this resampling procedure automatically accounts for variation in egg cohort sizes. In this case no subsampling is done within egg cohorts selected for a bootstrapped sample, because egg cohorts were usually constructed from all of the eggs laid by the adult females in a particular cage on a specific day.

\section{Analysis of the Initial Experiments}

In the first year of the study, egg cohorts were incubated at $10^{\circ} \mathrm{C}, 20^{\circ} \mathrm{C}, 25^{\circ} \mathrm{C}$, and $30^{\circ} \mathrm{C}$. Four temperature chambers were used, one for each temperature. Since no BLB eggs were observed to hatch at $10^{\circ} \mathrm{C}$, daily development rates were estimated only for the other three temperatures. The number of cohorts and number of individual BLB eggs maintained at each temperature are presented in Table 2. Based on the experience of the entomologists, values of $\mathrm{A}$ in formulas (4) and (5) were specified as 3, 5, and 9 days at $20^{\circ} \mathrm{C}, 25^{\circ} \mathrm{C}$, and $30^{\circ} \mathrm{C}$, respectively. No eggs were observed to hatch prior to the specified A for any of the respective temperatures, but increasing any of the A values would violate this criterion. It is possible to use smaller values of A. This would affect the estimates of $\delta$ and $\gamma$, but it would have relatively little affect on estimates of $\pi$ or M because large numbers of eggs were examined and final inspection times were used that allowed over $90 \%$ of the viable eggs to hatch.

Estimates of the probability of viable eggs $(\pi)$, the hatch time distribution parameters $(\delta$ and $\gamma$ ), and the median time to hatch for viable eggs (M), obtained by maximizing (3), are presented in Table 3 for each of the three temperatures. The estimates of $\pi$ increase as the temperature increases and are only slightly higher than the lower bounds provided by the observed proportions of hatched eggs reported in the last column of Table 2 . Estimates of the Weibull shape parameters are relatively unaffected by temperature. 
Estimates of the scale parameter and median hatch time decrease as temperature increases, indicating that eggs tend to hatch sooner and the variability in hatch times decreases as incubation temperature increases from $20^{\circ} \mathrm{C}$ to $30^{\circ} \mathrm{C}$.

Parameter estimates obtained by maximizing (3) for the Weibull model defined by (4) are listed in the third column of Table 3, and the fifth column gives estimates adjusted for bootstrap estimates of bias. The last column of Table 3 shows the bootstrap estimates of the standard deviations. For comparative purposes, the fourth column of Table 3 displays standard errors obtained from inverting the matrix of second partial derivatives of the IWM log-likelihood. Accounting for within cohort correlation with the bootstrap procedure increases the estimated standard errors by at least a factor of 1.9 in all cases.

The bootstrapped standard error for the median hatch time for viable BLB eggs at each temperature is computed by applying formula (5) to the estimates of $\delta$ and $\gamma$ from each of the 1000 bootstrap samples to obtain 1000 bootstrapped estimates of the median. The bootstrapped standard error is simply computed as the standard deviation of those 1000 bootstrapped estimates of the median. The standard error for the median reported in the fourth column of Table 3 is computed by applying the delta method to the covariance matrix for the estimates of $\delta$ and $\gamma$ obtained by inverting the negative of the matrix of estimated second partial derivatives of the IWM log-likelihood. Results for the inverse median can be computed in a similar fashion.

To further examine the effects of temperature on the proportions of viable BLB eggs, 1000 sets of estimates of the proportions of viable eggs at $20^{\circ} \mathrm{C}, 25^{\circ} \mathrm{C}$, and $30^{\circ} \mathrm{C}$ were constructed by randomly matching estimates from the three sets of bootstrapped samples. The method of least squares was used to fit a quadratic polynomial to each of the 1000 sets of estimates. The form of the model is

$$
\hat{\pi}=\beta_{0}+\beta_{1}(\mathrm{~T}-25)+\beta_{2}(\mathrm{~T}-25)^{2}+\epsilon,
$$

where $T=20,25,30$ represents temperature, $\hat{\pi}$ is the bootstrapped estimate of the proportion of viable eggs at the corresponding temperature, and $\epsilon$ is a random error. The mean and standard deviation of the resulting 1000 bootstrapped estimates for $\beta_{2}$ are 0.00000027 and 0.0011 , respectively, which suggests that the quadratic term is not needed. The formula for the linear regression of the proportion of viable BLB eggs on temperature is

$$
\hat{\pi}=0.742+0.0082(\mathrm{~T}-25), \quad \text { for } 20 \leq \mathrm{T} \leq 30 .
$$

The bootstrapped standard errors for the estimated intercept and slope shown in (7) are 0.012 and 0.00265 , respectively.

The same procedure was used to examine the effects of temperature on the inverse of the median hatch time for viable BLB eggs. Previous experience with beetle egg 
hatch times suggested that the percentage daily development rate, defined as the inverse of the median hatch time multiplied by $100 \%$, should be linearly related to temperature. The inverses of the bootstrapped estimates of the median hatch times were regressed on temperature for 1000 sets of bootstrapped estimates. The estimated regression formula is

$\frac{100 \%}{\text { Median hatch time }}=11.035+0.922(\mathrm{~T}-25), \quad 20 \leq \mathrm{T} \leq 30$.

The bootstrapped standard errors for the intercept and slope are 0.047 and 0.012 , respectively, indicating that both parameters are positive. Fitting a quadratic model to the inverses of the median hatch times resulted in an estimated coefficient of 0.00032 for the quadratic temperature term with a standard error of 0.0042 . Consequently, a straight line seems to adequately reflect the effect of temperature on the inverse median hatch time (development rate) for viable BLB eggs.

\section{The Need for Replication}

In the initial set of three experiments all egg cohorts incubated at a particular temperature were maintained in a single temperature chamber. Only three temperature chambers were used, one for each of the three temperatures. Consequently, bootstrap standard errors computed in the previous section essentially reflect only variation within and among cohorts within temperature chambers. Variation among replicated experiments for a single temperature done in different temperature chambers cannot be reliably assessed from these data, and such variation is not taken into account in the previous analyses of temperature effects on inverse medians and proportions of viable eggs. This was resolved by performing two additional replications of the experiment at $20^{\circ} \mathrm{C}, 25^{\circ} \mathrm{C}$, and $30^{\circ} \mathrm{C}$. In addition, sets of three replications were performed at $18^{\circ} \mathrm{C}$, $22^{\circ} \mathrm{C}, 28^{\circ} \mathrm{C}$, and $32^{\circ} \mathrm{C}$. Each of these experiments was performed in a different temperature chamber. These experiments were smaller than the original experiments, using fewer egg cohorts with about 20 eggs per cohort.

The number of cohorts, total number of eggs observed to hatch, and total number of eggs used in each experiment are listed in Table 4 . The fifth column of Table 4 gives the estimate of the proportion of viable eggs obtained from maximizing (3) with the Weibull survivor function defined by (4). Corresponding estimates of inverse median hatch times multiplied by $100 \%$, referred to as daily development rates, are listed in column 7 of Table 4 . Bootstrap estimates of standard errors of these estimates are presented in columns 6 and 8, respectively. The squares of the inverses of these standard errors were used as weights in weighted least squares estimation of regression models computed with the GLM procedure in the SAS computer package. 
The estimated proportions of viable eggs, from column 5 of Table 4, are plotted against temperature in Figure 1. The estimates from the original three experiments appear as solid dots. It appears that the linear trend suggested by these three points is a spurious result that could be attributed to temperature chamber variation. Weighted least squares regression applied to the points on this plot did not produce a significant slope $(\mathrm{p}$-value $=0.84)$. The R-squared value was 0.0021 . Between $18^{\circ} \mathrm{C}$ and $32^{\circ} \mathrm{C}$, there is no apparent affect of temperature on the viability of BLB eggs.

The percentage daily development rate of BLB eggs, given by the inverse of the median hatch time multiplied by $100 \%$, is affected by temperature. Estimates from column 7 on Table 4 are plotted against temperature in Figure 2. The points corresponding to the original three experiments are displayed with solid dots. Figure 2 also shows the weighted least squares estimate of a simple linear regression model. For this model, R-squared is 0.95 and the slope is significant (p-value $<.0001$ ). The estimated formula is

$\frac{100 \%}{\text { Median hatch time }}=10.699+0.871(\mathrm{~T}-25), \quad 18 \leq \mathrm{T} \leq 32$.

Adding quadratic and cubic terms to the model yields only a slight improvement in the $\mathrm{R}$-squared value from 0.940 to 0.967 , but both the quadratic term ( $\mathrm{p}$-value $=.0026$ ) and the cubic term ( $\mathrm{p}$-value $=.0031)$ are significant. This suggests that the accuracy of the straight line model begins to deteriorate outside of the temperature range from $20^{\circ} \mathrm{C}$ to $30^{\circ} \mathrm{C}$.

A referee noted that the weights used in the weighted least squares regression analysis are estimated which leads to underestimation of variability of parameter estimates. It is possible that a second application of a bootstrap or some other resampling procedure could be helpful, but this would greatly increase the computational burden and this suggestion has not yet been investigated.

\section{Summary}

This article presents a method for analyzing life event or survival data observed from cohorts of respondents where results for members of any single cohort can be correlated but members of different cohorts respond independently. This method does not require the specification of a joint survivor function for members of a single cohort, nor does it require the specification of a model for within cohort correlations, which are especially difficult to model when some members of a cohort may not be viable in the sense that the monitored life event never occurs. Only the form for a marginal distribution for survival or life event times for viable members of the overall population is required. In this application egg hatch times were either interval or right censored, but the bootstrap 
procedure also accommodates left censored and exact time data. Inspection schedules do not have to be the same for all cohorts and the procedure automatically accounts for variation in cohort sizes. An LFP approach was used to account for the possibility that some viable eggs may not be observed to hatch.

Bootstrap estimation was used to obtain appropriate estimates of covariance matrices for parameters estimated from a single experiment. This information was used in a weighted least regression analysis of the effects of temperature on the viability and development rates of BLB eggs. Replication of experiments in different temperature chambers revealed a spurious correlation between temperature and proportion of viable eggs suggested by the three original experiments.

Similar procedures can be used to model temperature effects on BLB larval development times. Ultimately, the results of these studies will be used to construct models for predicting peak emergence times and population sizes of F2 BLB adults from sample information on the time eggs are laid by the F1 generation. Combining this with predictions of the crop stage at the time of peak emergence of $\mathrm{F} 2$ adults will enable more cost effective and environmentally sound management strategies to be developed.

\section{Acknowledgements}

This research was supported by the National Cancer Institute, grant number 1 R01 CA51831-01A1, and the Iowa Agriculture and Home Economics Experiment Station, Ames, Iowa; Project Nos. 2580 and 3030. The BLB egg hatch experiments were performed by Michael R. Zeiss as part of his dissertation research at Iowa State University, under the direction of Professor Larry P. Pedigo, Department of Entomology.

\section{References}

Cox, D. R. and Oakes, D. (1984). Analysis of Survival Data. New York: Chapman Hall.

Efron, B. (1979). The Reitz Lecture. Bootstrap methods: another look at the jackknife. Annals of Statistics, 7, 1-26.

Efron, B. (1982). The Jackknife, the Bootstrap and Other Resampling Plans, Society for Industrial and Applied Mathematics, Philadelphia.

Herzog, D. C., Eastman, C. E. and Newsom, L. D. (1974). Laboratory rearing of the bean leaf beetle. Journal of Economic Entomology, 67, 794-795.

Huber, P. J. (1967). The behavior of maximum likelihood estimators under nonstandard conditions. Proceedings of the Fifth Berkeley Symposium, 221-233. 
Huster, W. J., Brookmeyer, R. and Self, S. G. (1989). Modelling paired survival data with covariates. Biometrics, 45, 145-156.

Kalbfleisch, J. D. and Prentice, R. L. (1980). The Statistical Analysis of Failure Time Data, New York: Wiley.

Koehler, J. K. and McGovern, P. G. (1990). Application of the LFP survival model to smoking cessation data. Statistics in Medicine, 9, 409-421.

Meeker, W. Q. (1987). Limited failure population life tests: applications to integrated circuit reliability. Technometrics, 29, 51-66.

Smelser, R. B. and Pedigo, L. P. (1991). Phenology of the bean leaf beetle, Cerotoma trifurcata (Foster), on soybean and alfalfa in central Iowa. Environmental Entomology, 20, 514-519.

Smelser, R. B. and Pedigo, L. P. (1992). Bean leaf beetle (Coleptera: Chrysomelidae) herbivory on leaf, stem, and pod components of soybean. Journal of Economic Entomology, 85, 2408-2412.

Turner, L. A. (1979). Bionomics of the bean leaf beetle, Cerotoma trifurcata (Foster) in Illinois. Unpublished Ph.D. dissertation, University of Illinois, Urbana-Champaign.

Table 1. Hatch results for 20 of the 111 bean leaf beetle egg cohorts incubated at $25^{\circ} \mathrm{C}$.

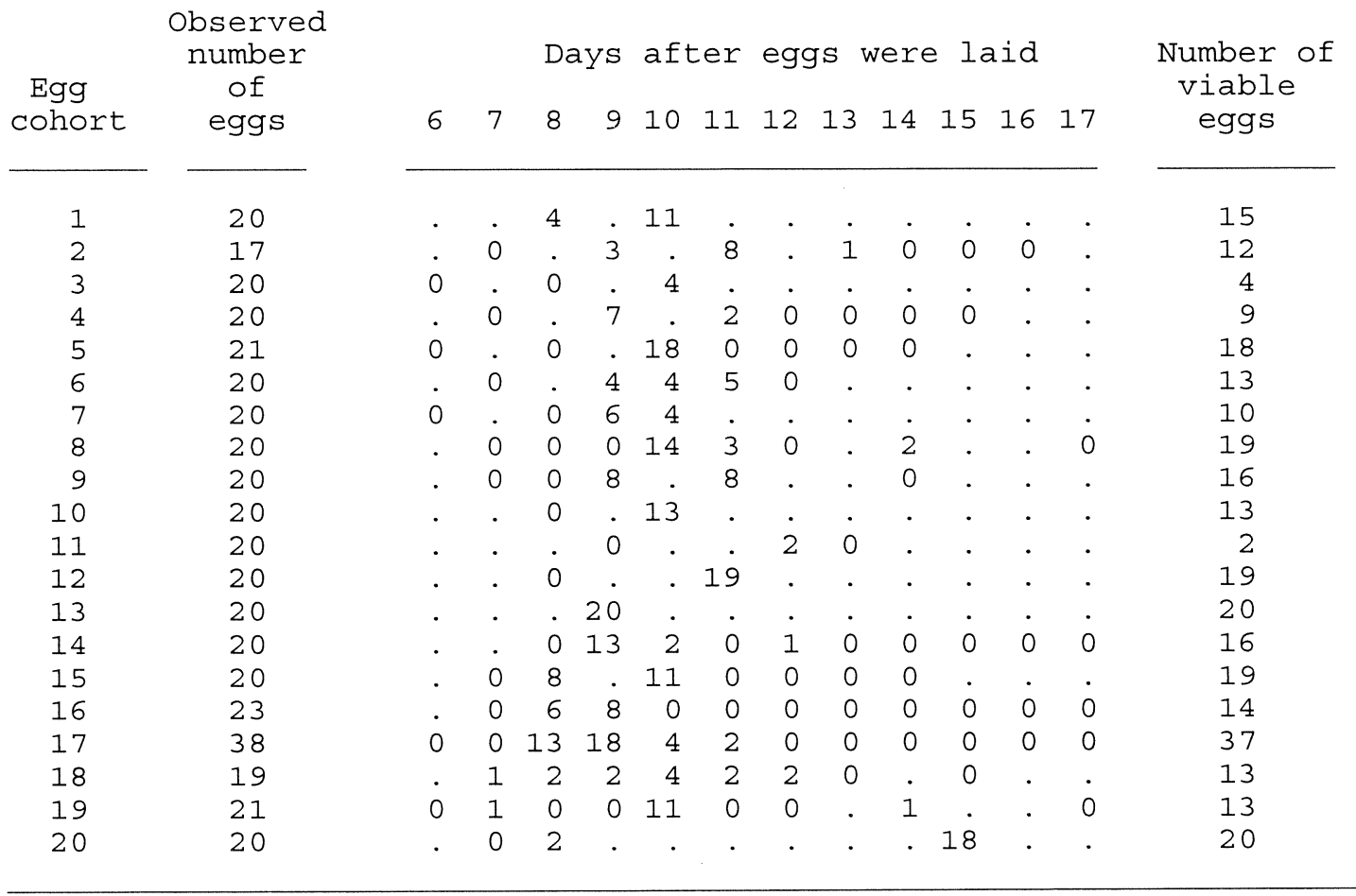


Table 2. Numbers of bean leaf beetle egg cohorts maintained at each temperature.

\begin{tabular}{|c|c|c|c|}
\hline Temperature & $\begin{array}{c}\text { Number } \\
\text { of } \\
\text { cohorts }\end{array}$ & $\begin{array}{c}\text { Number } \\
\text { of } \\
\text { eggs }\end{array}$ & $\begin{array}{l}\text { Percent } \\
\text { observed } \\
\text { to hatch }\end{array}$ \\
\hline $20^{\circ} \mathrm{C}$ & 150 & 2927 & 65.3 \\
\hline $25^{\circ} \mathrm{C}$ & 111 & 2150 & 71.6 \\
\hline $30^{\circ} \mathrm{C}$ & 127 & 2499 & 77.5 \\
\hline
\end{tabular}

Table 3. Estimates of parameters and standard errors for Weibull LFP models for BLB eggs incubated at three different temperatures.

\begin{tabular}{|c|c|c|c|c|}
\hline \multirow[b]{2}{*}{ Temperature } & \multirow{2}{*}{\multicolumn{2}{|c|}{$\begin{array}{c}\text { MLE's assuming } \\
\text { independent responses } \\
\text { Std. } \\
\text { Estimate error }\end{array}$}} & \multicolumn{2}{|c|}{$\begin{array}{l}\text { Bootstrap } \\
\text { estimates }\end{array}$} \\
\hline & & & Estimate & $\begin{array}{l}\text { std. } \\
\text { error }\end{array}$ \\
\hline scale $(\delta)$ & 7.172 & 0.048 & 7.167 & 0.144 \\
\hline Shape $(\gamma)$ & 3.804 & 0.076 & 3.830 & 0.220 \\
\hline Proportion viable $(\pi)$ & 0.700 & 0.009 & 0.701 & 0.020 \\
\hline Median hatch time (M) & 15.513 & 0.043 & 15.511 & 0.153 \\
\hline Scale $(\delta)$ & 4.486 & 0.036 & 4.485 & 0.098 \\
\hline Shape $(\gamma)$ & 3.832 & 0.082 & 3.871 & 0.259 \\
\hline Proportion viable $(\pi)$ & 0.742 & 0.010 & 0.742 & 0.024 \\
\hline Median hatch time (M) & 9.077 & 0.032 & 9.078 & 0.089 \\
\hline scale $(\delta)$ & 3.696 & 0.025 & 3.694 & 0.059 \\
\hline Shape $(\gamma)$ & 4.231 & 0.084 & 4.257 & 0.200 \\
\hline Proportion viable $(\pi)$ & 0.782 & 0.008 & 0.782 & 0.017 \\
\hline Median hatch time (M) & 6.389 & 0.028 & 6.389 & 0.054 \\
\hline
\end{tabular}


Table 4. Estimates of the proportion of viable eggs $(\pi)$ and the daily development rate $\left(\mathrm{M}^{-1} \times 100 \%\right)$ for $21 \mathrm{BLB}$ egg hatch experiments.

\begin{tabular}{|c|c|c|c|c|c|c|c|}
\hline $\begin{array}{l}\text { Temper- } \\
\text { ature } \\
\left({ }^{\circ} \mathrm{C}\right)\end{array}$ & $\begin{array}{l}\text { Number } \\
\text { of } \\
\text { cohorts }\end{array}$ & $\begin{array}{l}\text { Total } \\
\text { number } \\
\text { of } \\
\text { eggs }\end{array}$ & $\begin{array}{l}\text { Number } \\
\text { of eggs } \\
\text { observed } \\
\text { to hatch }\end{array}$ & $\begin{array}{c}\text { Estimated } \\
\text { Proportion } \\
\text { of viable } \\
\text { eggs }(\hat{\pi})\end{array}$ & $\begin{array}{c}\text { Standard } \\
\text { error of } \\
\hat{\pi}\end{array}$ & $\begin{array}{c}\text { Estimated } \\
\% \text { daily } \\
\text { development } \\
(1 / \hat{\mathrm{M}}) \\
\times 100 \%\end{array}$ & $\begin{array}{c}\text { Standard } \\
\text { error of } \\
(1 / \hat{\mathrm{M}}) \\
\times 100 \%\end{array}$ \\
\hline \multirow[t]{3}{*}{18} & 37 & 669 & 465 & 0.796 & 0.040 & 4.987 & 0.088 \\
\hline & 19 & 378 & 242 & 0.682 & 0.072 & 6.243 & 0.148 \\
\hline & 31 & 619 & 460 & 0.782 & 0.035 & 6.012 & 0.109 \\
\hline \multirow[t]{3}{*}{20} & 20 & 374 & 241 & 0.659 & 0.090 & 7.258 & 0.295 \\
\hline & 37 & 738 & 365 & 0.512 & 0.056 & 5.329 & 0.056 \\
\hline & 150 & 2929 & 1910 & 0.700 & 0.021 & 6.426 & 0.060 \\
\hline \multirow[t]{3}{*}{22} & 10 & 220 & 159 & 0.723 & 0.054 & 9.651 & 0.249 \\
\hline & 29 & 578 & 515 & 0.906 & 0.024 & 9.028 & 0.191 \\
\hline & 53 & 1008 & 364 & 0.599 & 0.070 & 7.350 & 0.126 \\
\hline \multirow[t]{3}{*}{25} & 85 & 1667 & 1362 & 0.818 & 0.024 & 10.13 & 0.086 \\
\hline & 15 & 301 & 238 & 0.791 & 0.060 & 12.37 & 0.450 \\
\hline & 111 & 2150 & 1539 & 0.743 & 0.025 & 11.03 & 0.104 \\
\hline \multirow[t]{3}{*}{28} & 27 & 447 & 314 & 0.757 & 0.053 & 14.29 & 0.251 \\
\hline & 19 & 348 & 220 & 0.640 & 0.064 & 15.12 & 0.216 \\
\hline & 17 & 341 & 266 & 0.786 & 0.042 & 14.66 & 0.337 \\
\hline \multirow[t]{3}{*}{30} & 127 & 2499 & 1937 & 0.782 & 0.019 & 15.65 & 0.133 \\
\hline & 22 & 444 & 314 & 0.708 & 0.039 & 15.53 & 0.258 \\
\hline & 22 & 444 & 313 & 0.705 & 0.037 & 16.04 & 0.226 \\
\hline \multirow[t]{3}{*}{32} & 34 & 612 & 358 & 0.586 & 0.044 & 16.70 & 0.256 \\
\hline & 27 & 633 & 354 & 0.556 & 0.050 & 15.46 & 0.278 \\
\hline & 14 & 236 & 219 & 0.928 & 0.029 & 15.72 & 0.231 \\
\hline
\end{tabular}




\section{Fig 1. Estimated Proportion of Viable Eggs}

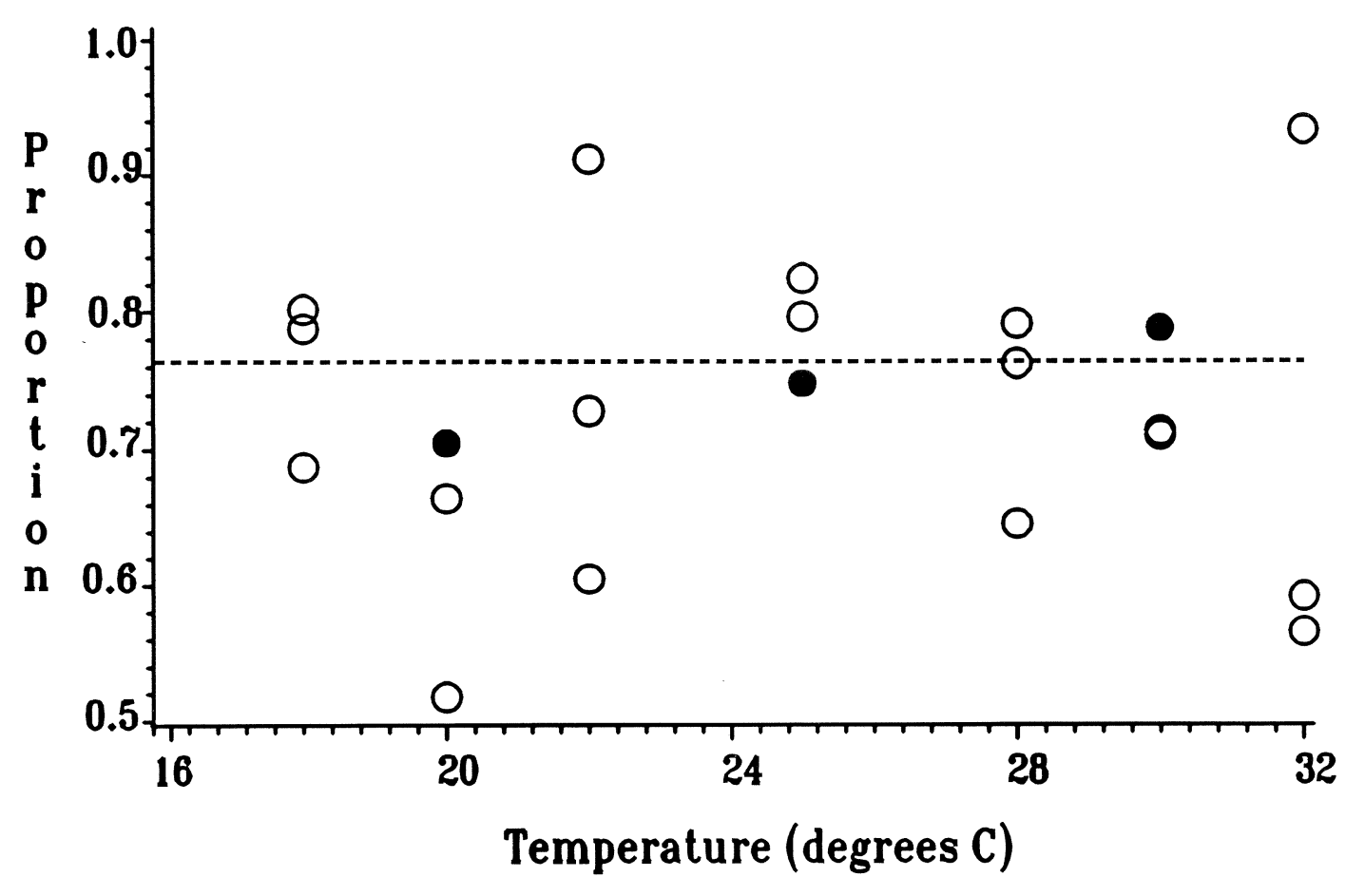


Fig 2. BLB Egg Daily Development Rates

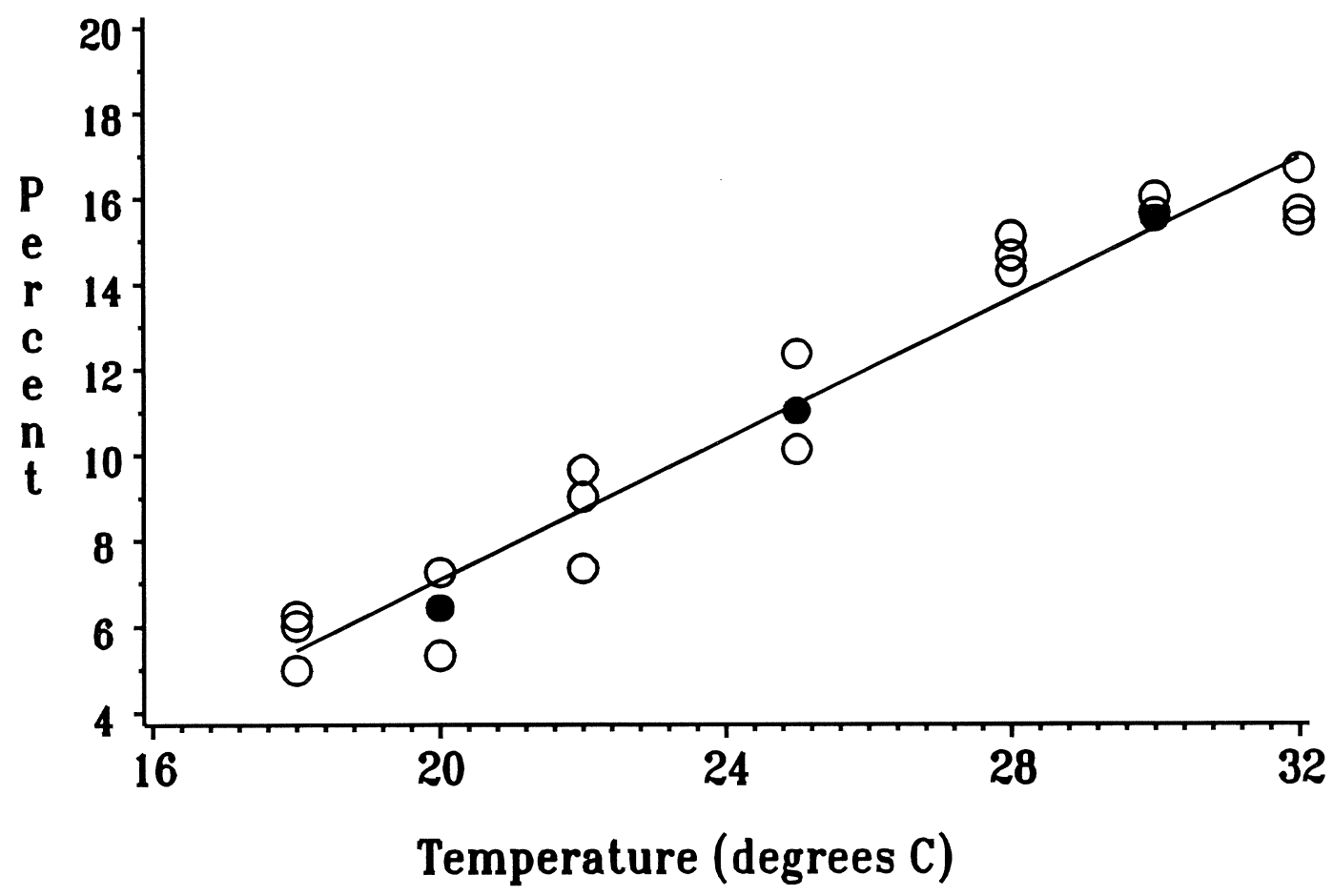

